\title{
Two-color nonlinear resonances in betatron oscillations of laser accelerated relativistic electrons
}

\author{
M. Lamač $\odot,{ }^{1,2, *}$ U. Chaulagain $\odot,{ }^{1}$ L. Jurkovičová $\odot,{ }^{1,3}$ J. Nejdl $\odot,{ }^{1}$ and S. V. Bulanov $\oplus^{1}$ \\ ${ }^{1}$ ELI Beamlines Center, Institute of Physics, Czech Academy of Sciences, Za Radnicí 835, 25241 Dolní Břežany, Czech Republic \\ ${ }^{2}$ Faculty of Mathematics and Physics, Charles University, Ke Karlovu 3, 12116 Prague, Czech Republic \\ ${ }^{3}$ Faculty of Nuclear Sciences and Physical Engineering, Czech Technical University, Břehová 78/7, 11519 Prague, Czech Republic
}

(Received 19 February 2021; accepted 15 June 2021; published 23 July 2021)

\begin{abstract}
X-ray radiation from laser wakefield accelerated (LWFA) electrons provides a collimated broadband femtosecond $\mathrm{x}$-ray source with micron-scale source size. However, the photon flux is still inadequate for a range of applications. By interaction of LWFA relativistic electrons with the second and the third harmonic of the laser driving the wakefield, we report more than an order of magnitude enhancement of photon flux emitted by the electrons undergoing betatron oscillations resonant with the harmonics. This phenomenon is demonstrated with analytical and numerical models, as well as particle-in-cell simulations.
\end{abstract}

DOI: 10.1103/PhysRevResearch.3.033088

\section{INTRODUCTION}

Laser wakefield acceleration (LWFA) has received considerable attention since its conception [1]. Recent experimental work reports $8 \mathrm{GeV}$ electron bunches being produced in a 20 -cm capillary plasma with a petawatt-class laser driver [2]. Betatron X-ray generation is connected to LWFA within the blowout regime $[3,4]$. The plasma cavity acts as a wiggler forcing electrons to oscillate and consequently radiate $\mathrm{X}$ rays known as betatron radiation $[5,6]$. The short duration of the x-ray pulses, small source size, synchronization with the driving laser, and all-optical compactness of the betatron $\mathrm{X}$-ray source provide a competitive alternative to conventional sources [7-9].

The applications of such ultrafast $\mathrm{x}$-ray sources range from high-resolution $\mathrm{x}$-ray imaging to ultrafast $\mathrm{x}$-ray science [9-15]. The hard $x$-ray source can be also employed in industrial applications such as X-ray computed tomography of dense objects [14]. An attempt on measurement of singleshot ultrafast x-ray absorption spectroscopy of a warm dense matter has been performed using this broadband $\mathrm{x}$-ray source; however, the photon flux is a limiting factor that prevents routine use of the source with single-shot measurements [15].

Features of betatron x-ray radiation depend on the electron Lorentz factor $\gamma$, betatron oscillation frequency $\omega_{\beta}=$ $\omega_{p} / \sqrt{2 \gamma}$, and betatron oscillation amplitude $r_{\beta}$, where $\omega_{p}=$ $\sqrt{n_{e} e^{2} / m_{e} \epsilon_{0}}$ is the electron plasma frequency, $n_{e}$ the electron density, $m_{e}$ the electron mass, $c$ the speed of light, $\epsilon_{0}$ the vacuum permittivity, and $e$ the elementary charge. We introduce the normalized transverse momentum amplitude

\footnotetext{
*marcel.lamac@eli-beams.eu
}

Published by the American Physical Society under the terms of the Creative Commons Attribution 4.0 International license. Further distribution of this work must maintain attribution to the author(s) and the published article's title, journal citation, and DOI. $\tilde{p}_{x}=p_{x} / m_{e} c$, known as undulator parameter $K[7,16,17]$, since $\tilde{p}_{x}=K=r_{\beta} k_{\beta} \gamma$, where $k_{\beta}$ is the betatron wave number. In the wiggler regime, where $K \gg 1$, the most important radiation parameters become the number of emitted photons per electron per oscillation period $N_{\gamma} \approx K / 30$ and the critical frequency of the synchrotron spectrum $\omega_{c}=\frac{3}{2} K \gamma^{2} \omega_{\beta}[7,18]$. We see that enhancement of transverse momentum is a crucial step toward achieving high-energy, high-flux x-ray radiation.

In recent years, many works demonstrating an increase of high-energy radiation emission from LWFA electrons have been published [19-25]. However, most of them report on generation of higher photon energy [19,20,22,24] with a limited number of works focusing on the photon flux enhancement of betatron $\mathrm{x}$-ray radiation in the kiloelectronvolt range $[21,25]$. Enhancement of betatron radiation photon flux in this energy range is critical for many applications such as single-shot time-resolved $\mathrm{x}$-ray absorption spectroscopy and ultrafast x-ray diffraction $[9,15]$.

In this paper, we show that the presence of the second harmonic (SH) and third harmonic (TH) of the fundamental laser frequency copropagating with the relativistic electrons enhance the photon flux of betatron $\mathrm{x}$-ray radiation by an order of magnitude. This enhancement originates from nonlinear resonances in betatron oscillations induced by two laser pulses with different frequencies interacting with the electrons. This resonance results in a boost of the transverse momentum $p_{x}$. The geometry of two-color betatron resonance is schematically shown in Fig. 1. The figure depicts a plasma cavity generated during the process of LWFA in a two-dimensional (2D) particle-in-cell (PIC) simulation, where a laser pulse with frequency $\omega_{d}$ drives a strong wakefield, causing selfinjected electrons to accelerate and oscillate within the plasma cavity. Betatron oscillation resonance with frequencies $\omega_{1}$ and $\omega_{2}$ is achieved when either of the shifted frequencies seen by the electron $\omega_{i}$ matches its betatron frequency $\omega_{i} \approx \omega_{\beta}$, where $\omega_{i}=\left(1-v_{z} / v_{p i}\right) \omega_{0 i}$ is the laser angular frequency seen by the electron, $i=1,2, \omega_{0 i}$ is the laser angular frequency, $v_{p i}$ is the phase velocity of the lasers, and $v_{z}$ is the longitudinal 


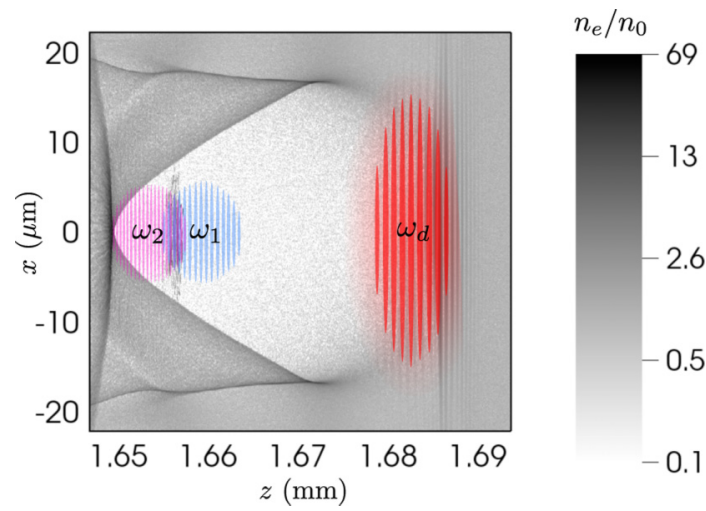

FIG. 1. Schematic of two-color betatron resonance. Normalized electron plasma density snapshot in grayscale from particle-in-cell (PIC) simulation of the two-color case presented in Sec. IV. Resonance is achieved due to the interaction of electrons with lasers of frequency $\omega_{1}$ and $\omega_{2}$. Laser with frequency $\omega_{d}$ drives laser wakefield acceleration (LWFA).

velocity of the electron. Such resonance results in significant transverse momentum enhancement [20,26,27].

This paper is organized as follows: In Sec. II, we show that, in the case of relativistic electrons close to dephasing under the paraxial approximation $p_{x} \ll p_{z}$, the driven electron oscillations can be described with the Duffing equation, which reveals the conditions for nonlinear resonances [28]. Section III shows solutions for full equations of motion for a more general case of a relativistic electron interacting with two lasers under the paraxial approximation, where the analytic resonance conditions from Sec. II are validated and the resonance dependency on plasma density $n_{e}$ is explored. Results from PIC simulations showing a presence of nonlinear resonances in the most general case, where laser and plasma evolution as well as acceleration are considered, are presented in Sec. IV. Analysis of emitted x-ray radiation from PIC results showing an order of magnitude enhancement in the case of a two-color scheme is presented in Sec. V. In Sec. VI, we propose an experimental setup capable of providing multicolor laser interaction in plasma targets based on our theoretical results with tunable laser polarizations and delays. Lastly, our results are summarized in the concluding Sec. VII.

\section{TWO-COLOR NONLINEAR RESONANCES IN PARAXIAL APPROXIMATION}

In this section, we derive the equation that reveals the resonances leading to transverse momentum enhancement present in relativistic electrons oscillating in a plasma channel while interacting with two laser frequencies. The equations of motion of an electron are

$$
\begin{aligned}
& \frac{d \mathbf{p}}{d t}=-e(\mathbf{E}+\mathbf{v} \times \mathbf{B}), \\
& \frac{d \mathbf{r}}{d t}=\mathbf{v}=\frac{\mathbf{p}}{m_{e} \gamma} .
\end{aligned}
$$

The electromagnetic fields of the plasma cavity in the cylindrical coordinate system $(r, \theta, z)$, where $r=\sqrt{x^{2}+y^{2}}$ is the radial coordinate, are given by the longitudinal electric field $E_{z} / E_{w b}=k_{p} \xi / 2$, radial electric field $E_{r} / E_{w b}=k_{p} r / 4$, and azimuthal magnetic field $c B_{\theta} / E_{w b}=-k_{p} r / 4$, where $\xi=z-v_{\phi} t$ is the wake comoving variable, $v_{\phi}$ is the phase velocity of the wake, $k_{p}$ is the plasma wave number, and $E_{w b}=m \omega_{p} c / e$ is the cold nonrelativistic wave-breaking field [4,29-31]. The equations of motion for an electron in such a cavity without any additional fields are then $\dot{\mathbf{p}}=-e(\mathbf{E}+\mathbf{v} \times \mathbf{B})=\mathbf{F}_{\|}+\mathbf{F}_{\perp} \approx-m \omega_{p}^{2}\left(\xi \mathbf{e}_{z} / 2+r \mathbf{e}_{r} / 2\right)$, where the last expression assumes $p_{\perp} / p_{z} \ll 1$ [7]. Furthermore, for electrons near the dephasing point $\xi \approx 0$, we only have the radial component of the force $\dot{\mathbf{p}} \approx-m \omega_{p}^{2} \mathbf{e}_{r} r / 2$. The laser field in the plane-wave approximation is given as $E_{\text {laser }}=E_{1} \sin \left(\phi_{1}\right)+E_{2} \sin \left(\phi_{2}\right)$, and the corresponding magnetic field is given as $B_{\text {laser }}=\left(E_{1} / v_{p 1}\right) \sin \left(\phi_{1}\right)+\left(E_{2} / v_{p 2}\right) \sin \left(\phi_{2}\right)$, where $E_{i}$ is the electric intensity amplitude, $\phi_{i}=\omega_{0 i}\left(t-z / v_{p i}\right)+\theta_{i}$ is the laser phase, and $\theta_{i}$ is the initial phase, $i=1,2$. The laser field is linearly polarized in the $x$ direction. By restricting the electron to planar motion $\left[p_{y}(t)=0\right]$, the momentum Eqs. (1) and (2), with both the cavity and laser fields included, become

$$
\begin{aligned}
& \frac{d p_{x}}{d t}=-e\left[\eta_{1} E_{1} \sin \left(\phi_{1}\right)+\eta_{2} E_{2} \sin \left(\phi_{2}\right)\right]-\frac{m_{e} \omega_{p}^{2}}{2} x, \\
& \frac{d p_{z}}{d t}=-e v_{x}\left[\frac{E_{1}}{v_{p 1}} \sin \left(\phi_{1}\right)+\frac{E_{2}}{v_{p 2}} \sin \left(\phi_{2}\right)\right],
\end{aligned}
$$

where the factor $\eta_{i}=1-v_{z} / v_{p i}$ comes from the sum of the electric field and vector product of velocity and magnetic field in Eq. (1). Taking the time derivative of Eq. (3) with the use of normalized transverse momentum $\tilde{p}=p / m_{e} c$ gives us the following equation for a nonlinear oscillator:

$$
\frac{d^{2} \tilde{p}_{x}}{d t^{2}}+\omega_{\beta}^{2} \tilde{p}_{x}=\tilde{a}_{1} \cos \left(\phi_{1}\right)+\tilde{a}_{2} \cos \left(\phi_{2}\right),
$$

where $\tilde{a}_{i}=a_{i} \omega_{i}^{2}$, and $a_{i}=e E_{i} /\left(m c \omega_{0 i}\right)$ is the normalized laser field amplitude, $i=1,2$. By excluding the electrostatic longitudinal wakefield generated during LWFA in Eqs. (1) and (2), we restrict ourselves to the case of wakefield pre-accelerated relativistic electrons with $\gamma \gg 1$ close to dephasing, at which point the wakefield contribution is negligible. This description is also applicable for direct laser accelerated electrons oscillating in self-generated transverse quasistatic fields of plasma channels generated by long laser pulses with relativistic intensities [26,32], generalized to the case when two laser frequencies are present. We are interested in the dynamics of LWFA ultrarelativistic oscillating electrons for which the paraxial approximation $p_{z} \gg p_{x}$ with $v_{z} \approx c$ is valid, and we have $p_{x} / p_{z} \approx v_{x} / c \ll 1$; therefore, $v_{x} \ll c<$ $v_{p i}$, which means that the contribution from the magnetic field to the transverse momentum $p_{x}$ through $p_{z}$ is minor compared with the electrical field contribution in Eq. (3). In other words, variables $p_{x}$ and $x$ are oscillating fast compared with slowly varying variables $v_{z}$ and $\gamma$ [32]. It is therefore reasonable to assume for slow variables that $\dot{\gamma}, \dot{v}_{z}, \dot{p}_{z} \approx 0$ on a suitable timescale, and therefore, $p_{z}=p_{z}(0)=: p_{z 0}$ on the timescale of betatron oscillations $\sim \omega_{\beta}^{-1}$. The Lorentz factor can be then 
approximated as

$$
\begin{aligned}
\gamma & =\sqrt{1+\tilde{p}_{x}^{2}+\tilde{p}_{z 0}^{2}}=\sqrt{1+\tilde{p}_{z 0}^{2}} \sqrt{1+\frac{\tilde{p}_{x}^{2}}{1+\tilde{p}_{z 0}^{2}}} \\
& \approx \gamma_{0}\left(1+\frac{\tilde{p}_{x}^{2}}{2 \gamma_{0}^{2}}\right),
\end{aligned}
$$

where $\gamma_{0}=\sqrt{1+\tilde{p}_{z 0}^{2}}$, which is the initial Lorentz factor, and since we are considering pre-accelerated relativistic electrons also, $\gamma_{0} \gg 1$. Further on, we have

$$
\omega_{\beta}^{2}=\frac{\omega_{p}^{2}}{2 \gamma} \approx \frac{\omega_{p}^{2}}{2 \gamma_{0}}\left(1-\frac{\tilde{p}_{x}^{2}}{2 \gamma_{0}^{2}}\right)=\omega_{\beta_{0}}^{2}\left(1-\frac{\tilde{p}_{x}^{2}}{2 \gamma_{0}^{2}}\right) .
$$

The paraxial approximation $p_{x} \ll p_{z}$ with $v_{z} \approx c$ further allows us to approximate frequency seen by electrons as $\dot{\phi}_{i}=\left(1-v_{z} / v_{p i}\right) \omega_{0 i}=\eta_{i} \omega_{0 i}=\omega_{i} \approx$ const.; therefore, the laser phase satisfies $\phi_{i}(t) \approx \omega_{i} t+\theta_{i}$. We note that the relative laser phase $\left(\theta_{2}-\theta_{1}\right)$ influences the detailed structure of simultaneous resonances, as discussed in Ref. [28]; however, the maximum attainable transverse momentum amplitude, which we are interested in, remains the same for all relative phase shifts. Therefore, we do not concern ourselves further with this parameter and, for brevity, set $\theta_{2}=\theta_{1}=0$. Plugging Eq. (8) into Eq. (5) gives

$$
\frac{d^{2} \tilde{p}_{x}}{d t^{2}}+\omega_{\beta_{0}}^{2} \tilde{p}_{x}-\frac{\omega_{\beta_{0}}^{2}}{2 \gamma_{0}^{2}} \tilde{p}_{x}^{3}=\sum_{i=1}^{2} \tilde{a}_{i} \cos \left(\omega_{i} t\right) .
$$

Equation (9) has the form of the Duffing equation with two-frequency forcing [28]. We will summarize the most important consequences of nonlinear Eq. (9), as discussed in Ref. [28]. We see that the nonlinearity coefficient of $\tilde{p}_{x}^{3}$ in Eq. (9), i.e., $-\omega_{\beta_{0}}^{2} / 2 \gamma_{0}^{2}$, has a negative sign, indicating a softening nonlinearity of the system, which means that the multivalued amplitude-frequency response curve bends toward frequencies below $\omega_{i} \approx \omega_{\beta_{0}}$. For a fixed $\gamma_{0}$, the nonlinearity changes only with electron density $n_{e}$. In addition, nonlinearity in Eq. (9) allows for multiple nonlinear resonances apart from the primary resonance $\omega_{i} \approx \omega_{\beta_{0}}$. The condition for these resonances can be generally given as

$$
\omega_{\beta_{0}} \approx \frac{m}{n} \omega_{i},
$$

where $m$ and $n$ are natural numbers, $i=1,2$, and either $m=n=1$ (primary resonance), $m=1, n \neq 1$ (subharmonic resonances), or $n=1, m \neq 1$ (superharmonic resonances). The strengths and widths of the frequency ranges in which these resonances occur decrease dramatically for large $m, n$; therefore, only small values of $m, n$ are usually observed $[28,33]$. We note that harmonic resonances in Eq. (10) arise even in single-frequency-driven nonlinear oscillations. Multifrequency forcing, however, generates additional combination resonances in nonlinear oscillations. The principal combination resonances are given by the following conditions:

$$
\begin{aligned}
& \omega_{\beta_{0}} \approx\left| \pm 2 \omega_{i} \pm \omega_{j}\right|, \\
& \omega_{\beta_{0}} \approx \frac{1}{2}\left(\omega_{i} \pm \omega_{j}\right),
\end{aligned}
$$

where $i, j=1,2$ [28]. The presence of two forcing terms also enables simultaneous resonances to occur, i.e., for given frequencies $\omega_{i}$, the resonance conditions in Eqs. (10)-(12) may overlap.

The main physical mechanism behind transverse momentum enhancement in two-color forced oscillations is therefore twofold. First, we have superposed primary, subharmonic, and superharmonic resonances from respective laser fields given by Eq. (10). Second, we obtain the combination resonances Eqs. (11) and (12), which are unique to multifrequency forcing, further enriching the range of possible resonances as well as enabling simultaneous resonances for oscillating electrons. The immediate consequence of the presence of such resonances in electron oscillations is the enhancement of betatron $\mathrm{X}$-ray radiation due to transverse momentum enhancement, as it is discussed in Sec. I.

We note that this model of forced betatron oscillations of relativistic electrons close to dephasing with $\gamma \gg 1$ is qualitatively valid, even when weak longitudinal electrostatic wakefield is included in the equations of motion in Eqs. (1) and (2). Such inclusion causes the decrease of the betatron frequency $\omega_{\beta} \approx \gamma^{-1 / 2}$ and slow increase of the strength parameter $K \approx \gamma^{1 / 4}$ due to electron energy gain $\sim \gamma$ on a timescale much longer than the timescale of betatron oscillations $\tau_{\beta} \approx \omega_{\beta}^{-1}$ [7]. To be precise, our description is valid for slowly varying betatron frequency satisfying the condition $\omega_{\beta}^{2} \gg\left|\dot{\omega}_{\beta}\right| \approx 0$, for which the nonlinear oscillator model is not affected by the negligible betatron frequency change. The previous assumption is further validated for relativistic electrons close to dephasing in Sec. IV, where it is also shown that the conditions for transverse momentum resonance hold even in full PIC simulations.

\section{SINGLE-PARTICLE SIMULATIONS}

The equations of motion in Eqs. (1) and (2), for a relativistic electron oscillating in plasma cavity with a presence of one or two laser fields in the paraxial approximation, were solved numerically using the fourth-order Runge-Kutta scheme of numerical integration with time step small enough to achieve numerical convergence. The calculation is simplified considerably due to the paraxial approximation $p_{x} \ll p_{z}, v_{z} \approx c$, which allows us to consider the laser phase as $\phi_{i}(t) \approx \omega_{i} t$. Note that, here, we do not consider the approximation $\dot{p}_{z} \approx 0$ as in Sec. II. The structure of resonances does not depend on the choice of initial transverse momentum [28], and therefore, we set it for all cases as $\tilde{p}_{x}(0)=0$. We neglect the presence of the longitudinal wakefield since we consider relativistic electrons close to dephasing, as discussed in Sec. II. We set field strengths as $a_{1} \approx a_{2} \approx 1$, corresponding to experimentally accessible values for harmonics of the driving laser. We consider both cases of single and two-color configuration. Lastly, we conduct simulations for a large range of plasma densities beyond the $n_{e} \approx 10^{19} \mathrm{~cm}^{-3}$ that is usually used for betatron $\mathrm{x}$-ray generation to reveal the structure of resonances even in the strongly nonlinear regime.

In the single-color case, a relativistic electron interacts only with a single laser field with $\omega_{1}$, which corresponds to the schematic in Fig. 1 when laser with $\omega_{2}$ is not present. In Figs. 2(a) and 2(b), we show the frequency response of 

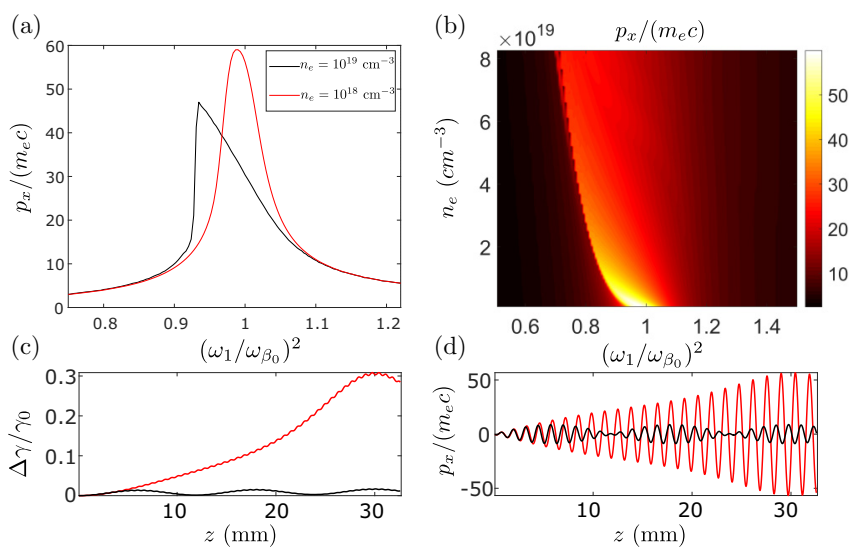

FIG. 2. Single-color case. (a) Frequency response of $\tilde{p}_{x}$ for $p_{x}(0)=0$. Nonlinearity emerges for higher values of electron plasma density $n_{e}$. For $n_{e}=5 \times 10^{18} \mathrm{~cm}^{-3}$ (red), we see linear response for resonance conditions in the range $\left(\omega_{1} / \omega_{\beta_{0}}\right)^{2} \in(0.8,1.2)$ corresponding to initial energies $\gamma_{0} \in(1.5,2.5) \times 10^{4}$, and we see strong nonlinear response for higher density $n_{e}=5 \times 10^{19} \mathrm{~cm}^{-3}$ (black) for $\left(\omega_{1} / \omega_{\beta_{0}}\right)^{2} \in(0.8,1.2)$ corresponding to $\gamma_{0} \in(1.5,2.5) \times 10^{3}$. (b) Frequency response of $\tilde{p}_{x}$ for varying $n_{e}$. (c) Normalized energy gain and (d) transverse momentum evolution for $n_{e}=5 \times 10^{18} \mathrm{~cm}^{-3}$, $\left(\omega_{1} / \omega_{\beta_{0}}\right)^{2}=0.8, \gamma_{0}=3.2 \times 10^{3}$ (black), and $\left(\omega_{1} / \omega_{\beta_{0}}\right)^{2}=0.9, \gamma_{0}=$ $3.6 \times 10^{3}$ (red). The nonshifted laser wavelength is $\lambda_{1}=404 \mathrm{~nm}$.

normalized transverse momentum amplitude, which is the maximum transverse momentum an electron achieves during periodic resonant oscillations. The frequency ratio $\omega_{1} / \omega_{\beta_{0}}$ is tuned by varying the initial electron energy since, for a fixed laser frequency and density, we have $\left(\omega_{1} / \omega_{\beta_{0}}\right)^{2} \approx \gamma_{0}$. We observe the emergence of nonlinear response for increasing plasma density $n_{e}$ featuring up-jump discontinuity and the bending of the frequency response curve as predicted by Eq. (9). We note that the initial condition $\tilde{p}_{x}(0)=0$ leads to the lower branch of the resonance curve for frequencies smaller than that corresponding to the up-jump discontinuity [28]. Larger initial momenta $p_{x}(0) \neq 0$ may lead to upperbranch amplitudes producing larger transverse momentum amplitudes left of the jump discontinuity, as is usual in multivariate frequency responses of nonlinear oscillators. The structure of the resonances, however, does not depend on the initial condition as previously noted. Figures 2(c) and 2(d) show that, within the paraxial approximation, resonant interaction leads to substantial energy and normalized transverse momentum gain even in the single-color case.

In the two-color case, two laser fields are interacting with electrons, as shown schematically in Fig. 1. The frequency response of the normalized transverse momentum amplitude with varying electron plasma density is shown in Fig. 3(a). We see two primary resonance regions due to $\omega_{1}$ and $\omega_{2}$. We also see that increasing density leads to stronger nonlinearity, as predicted by Eq. (9). For high values of electron plasma density $n_{e}$, the emergence of harmonic and simultaneous resonances creates detached regions of large transverse momentum. Figure 3(b) shows a strongly nonlinear region with $n_{e}>5 \times 10^{19} \mathrm{~cm}^{-3}$, where complex structures of simultaneous, combination, and harmonic resonances emerge. We
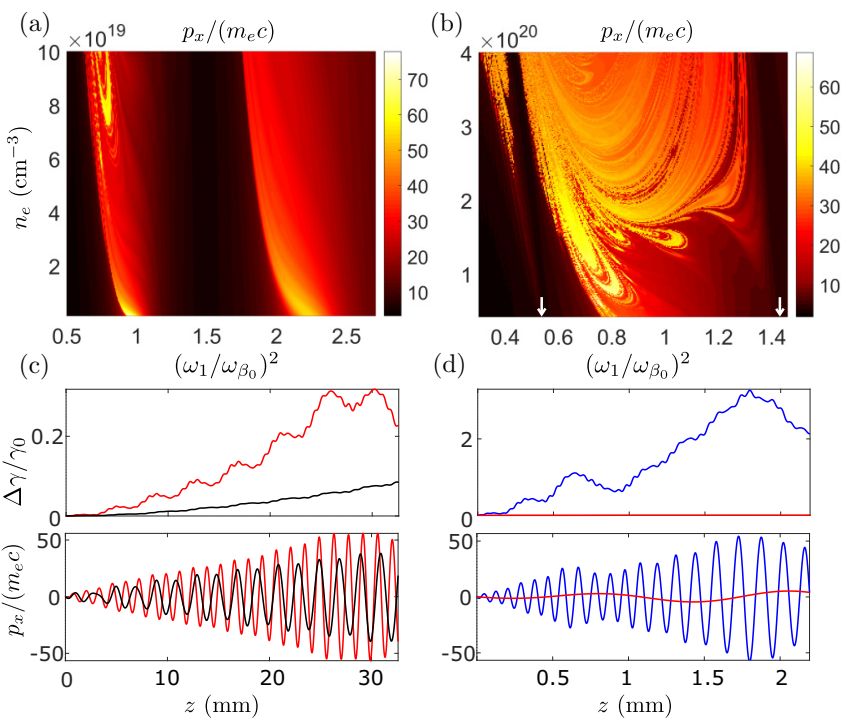

(d)

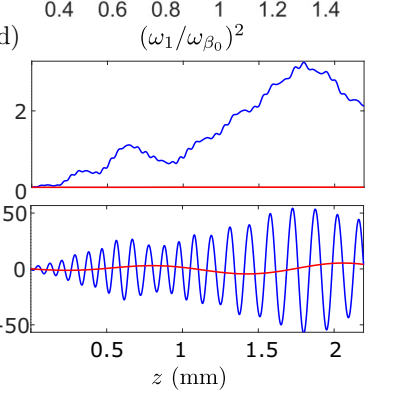

FIG. 3. Two-color case. (a) Frequency response of $\tilde{p}_{x}$ for $p_{x}(0)=0$ with varying $n_{e}$. (b) The strongly nonlinear region for $n_{e}>5 \times 10^{19} \mathrm{~cm}^{-3}$. (c) Normalized energy gain and (d) transverse momentum evolution for $n_{e}=5 \times 10^{18} \mathrm{~cm}^{-3},\left(\omega_{1} / \omega_{\beta_{0}}\right)^{2}=0.9, \gamma_{0}=$ $3.6 \times 10^{3}$ (red), and $\left(\omega_{1} / \omega_{\beta_{0}}\right)^{2}=2.1, \gamma_{0}=8.6 \times 10^{3}$ (black), and for $n_{e}=8 \times 10^{19} \mathrm{~cm}^{-3},\left(\omega_{1} / \omega_{\beta_{0}}\right)^{2}=0.8, \gamma_{0}=2 \times 10^{2}$ (blue). White arrows indicate emergence of nonlinear resonances $\left(\omega_{1} / \omega_{\beta_{0}}\right)^{2}=$ $0.5625,1.44$. The nonshifted laser wavelengths are $\lambda_{1}=404 \mathrm{~nm}$ and $\lambda_{2}=269 \mathrm{~nm}$.

indicate [white arrows in Fig. 3(b)] emergence of some of the nonlinear resonances predicted by Eqs. (10)-(12). For example, since the shifted frequency of the TH is $\omega_{2} \approx\left(\frac{2}{3}\right) \omega_{1}$, Eq. (12) gives combination resonance $\left[\omega_{1}+\left(\frac{2}{3}\right) \omega_{1}\right] / 2=\omega_{\beta_{0}}$; therefore, $\left(\omega_{1} / \omega_{\beta_{0}}\right)^{2} \approx 1.44$ (white arrow on the right). An interesting feature seen in Fig. 3(a) is that, since for a fixed electron density $n_{e}$ we have $\left(\omega_{i} / \omega_{\beta_{0}}\right)^{2} \approx \gamma_{0}$, nonlinearity is strongly suppressed in the primary resonance region from $\omega_{2}$, which is positioned at $\left(\omega_{1} / \omega_{\beta_{0}}\right)^{2}=2.25$. This is predicted by the inverse cube dependence of the nonlinearity coefficient on high initial electron energy in Eq. (9), i.e., $\omega_{\beta_{0}}^{2} / 2 \gamma_{0}^{2}=$ $\omega_{p}^{2} / 4 \gamma_{0}^{3}$. Figures 3(c) and 3(d) show selected resonant evolutions. We draw attention to the benefit of employment of higher frequency lasers, in this case $\omega_{2}$, since they induce otherwise unattainable primary resonance for electrons accelerated through LWFA to high energies $\gamma_{0} \approx\left(\omega_{2} / \omega_{\beta_{0}}\right)^{2}$, boosting their $\tilde{p}_{x}$.

Single-particle simulations reveal the structure of the resonances present in betatron oscillations of relativistic electrons close to dephasing when interacting with a two-color laser field in the paraxial approximation. We have shown the emergence of nonlinear resonances in Eqs. (10)-(12) predicted by Eq. (9). We note that single-particle simulations neglect the spatial and temporal profile of the laser pulses as well as its self-focusing and depletion or the presence of accelerating longitudinal plasma wakefield and its evolution. To account for full dynamics, we conducted PIC simulations described in the following section. 


\section{PIC SIMULATIONS}

To confirm the presence of two-color nonlinear resonances beyond the paraxial approximation in full detail throughout the LWFA process, we performed 2D PIC simulations within the fully relativistic, massively parallelized PIC code $\mathrm{EPOCH}$ [34]. The approximation of 2D geometry is reasonable since all lasers are set with the same linear polarization in the $x$ direction within the $x-z$ plane of the simulation, which forces the dynamics of the electron oscillations and the associated resonances induced by the interacting lasers into a 2D plane. The second-order Yee scheme Maxwell solver was used in the PIC simulations. To reduce numerical dispersion in the second-order scheme, the Courant-Friedrichs-Lewy number was set as $c \Delta t / \Delta z=0.99$. The moving window simulation box was set as $100 \times 70 \mu \mathrm{m}$ with grid size $6000 \times 1000$, which corresponds to 16 cells per wavelength for the shortest laser wavelength in the simulation $\lambda_{2}=269 \mathrm{~nm}$, ensuring proper resolution of all wavelengths involved. Furthermore, thirdorder interpolation of fields to particles was used to further suppress numerical heating and time-staggering error $[35,36]$. The number of macro-particles per cell was two. The lasers were focused upon the entrance into the 5-mm-long homogeneous pre-ionized helium gas target with smooth edges and plateau value of electron plasma density $n_{e}=4 \times 10^{18} \mathrm{~cm}^{-3}$. Such gas targets are routinely used in plasma betatron $\mathrm{x}$-ray sources $[6,17,21]$. The value of plasma density was selected this low to generate a larger bubble and therefore prevent interaction of the accelerating electrons with the laser tail of the LWFA-driving frequency $\omega_{d}$. To simplify the analysis, we focus purely on the dynamics of the electrons and the laser. We therefore neglect collisions, ionization, or quantum electrodynamics effects, although we note that interference effects can play a role in two-color laser ionization injection [34,37,38].

In the nonresonant case, only the LWFA-driving laser with $\omega_{d}$ shown in Fig. 1 is present with $\lambda_{0}=808 \mathrm{~nm}$. Laser strength was set as $a_{0}=5.3$, full width at half maximum (FWHM) pulse duration $\tau_{0}=28 \mathrm{fs}$, and waist diameter $d_{0}=$ $28 \mu \mathrm{m}$. This case corresponds to standard LWFA and betatron $\mathrm{X}$-ray generation since the laser does not interact with the accelerating electrons due to low plasma density and consequently large bubble radius.

In the single-color case, the driving laser was set as in the previous case. The SH was set with $\lambda_{1}=404 \mathrm{~nm}, a_{1}=$ $1.2, \tau_{1}=56 \mathrm{fs}$, and $d_{1}=10 \mu \mathrm{m}$. To place the peak of the $\mathrm{SH}$ in the interaction region where electrons oscillate, the peakto-peak time delay between the two pulses was set to $100 \mathrm{fs}$. The time delay was set to place the $\mathrm{SH}$ in the interaction region where electrons accelerate without overlapping with the electron bubble sheath. This case corresponds to single-color betatron resonance since the driving laser does not interact with the accelerating electrons, and resonant oscillations are induced by the $\mathrm{SH} \omega_{1}$. In terms of the schematic in Fig. 1, the laser corresponding to frequency $\omega_{2}$ is not present.

In the two-color case, the driving laser was as in the previous cases. The $\mathrm{SH}$ had slightly shorter pulse duration with $\lambda_{1}=404 \mathrm{~nm}, a_{1}=1.2, \tau_{1}=42 \mathrm{fs}$, and $d_{1}=10 \mu \mathrm{m}$ to compensate for frequency conversion into the TH. The peakto-peak time delay to the LWFA-driving laser was $92 \mathrm{fs}$.
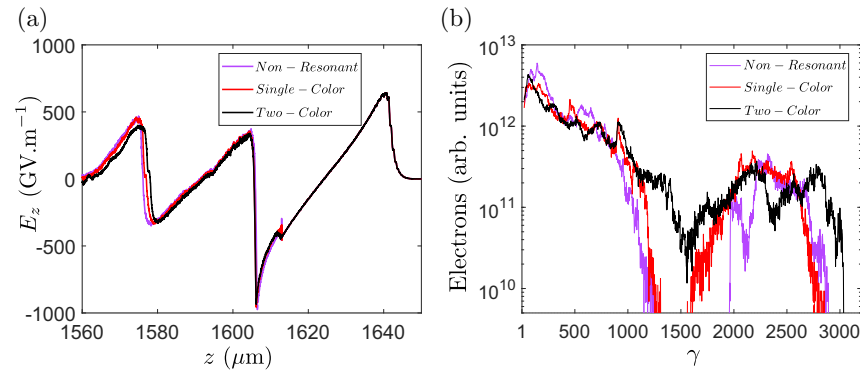

FIG. 4. (a) Longitudinal electrostatic wakefields at $1.6 \mathrm{~mm}$ inside the gas target for all three cases. (b) Electron spectra at gas target exit at $5 \mathrm{~mm}$.

The TH was also present with $\lambda_{2}=269 \mathrm{~nm}, a_{2}=0.7, \tau_{2}=$ $56 \mathrm{fs}$, and $d_{2}=10 \mu \mathrm{m}$, and the peak-to-peak time delay to the fundamental was $100 \mathrm{fs}$. This case corresponds to single-color betatron resonance since the driving laser does not interact with the accelerating electrons, and resonant oscillations are induced by the $\mathrm{SH} \omega_{1}$. In terms of the schematic in Fig. 1, the laser corresponding to frequency $\omega_{2}$ is not present. We note that the field of the harmonics interacting with the electrons is kept stable due to the plasma cavity generated by the main driver with $\omega_{d}$. The high-density electron sheath of the cavity acts as a waveguide for the small amplitude harmonics, which would otherwise diffract in plasma or vacuum over few Rayleigh lengths.

The total number of accelerated electrons is comparable in all three cases. This is understandable since the SH with $a_{1}=1.2$ does not overlap with the electron plasma sheath, and the TH with peak strength $a_{2}=0.7$ overlaps with the sheath only in the region where $a_{2} \approx 0.3$, which means that the ponderomotive force $\sim \nabla a^{2}$ has negligible effect on the evolution of the wake driven by the fundamental harmonic with $a_{0}=5.3$. In Fig. 4(a), we show that the accelerating wakefields for all cases vary $<5 \%$ at $1.6 \mathrm{~mm}$ inside the target, where the first injected electron bunches are accelerated.

It is also important to consider possible dimensional effects of the wake evolution. It has been shown that, in 2D simulations, the wakefield plasma bubble is elongated, and the focusing fields are stronger than in the case of threedimensional (3D) simulations of plasma wakefield, while the structure of the accelerating field inside the cavity remains

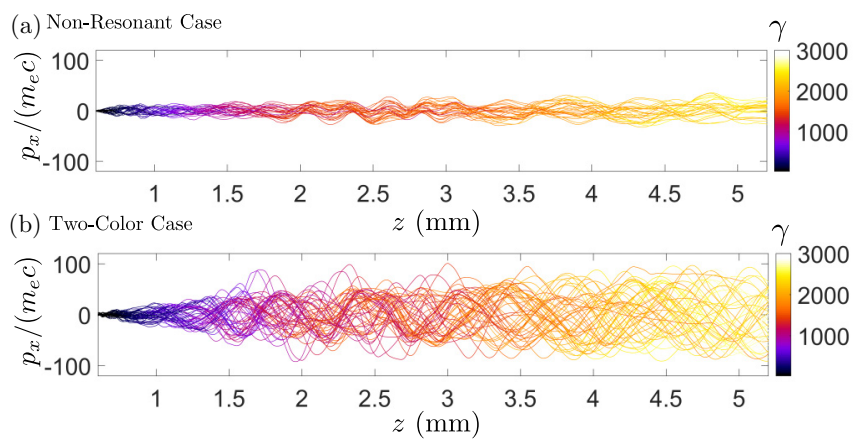

FIG. 5. $\tilde{p}_{x}$ and $\gamma$ evolutions for electrons that reach $\gamma>1800$. (a) Nonresonant case, where no electrons are interacting with a laser. (b) Two-color case. 

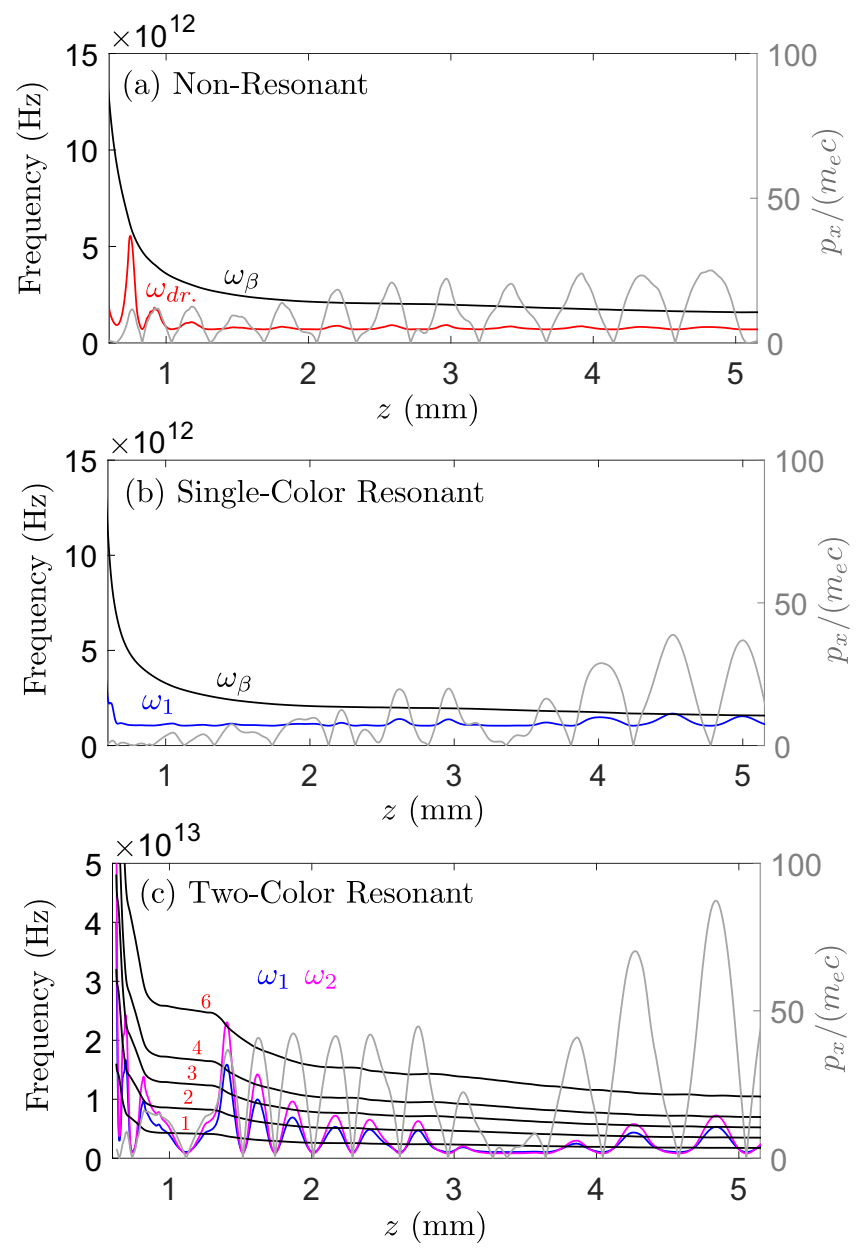

FIG. 6. Shifted laser frequencies $\omega_{1,2}$ seen by a representative electron (left axis, colored), betatron frequency harmonics $n \omega_{\beta}$ (left axis, black, orders highlighted by red number), and absolute value of normalized transverse momentum $p_{x} / m_{e} c$ (right axis, gray).

unchanged in both cases [39]. The reduction of the bubble in the realistic 3D case can be simply compensated by changing group delays of the harmonics with respect to the LWFA driver. Weaker focusing fields in 3D geometry also imply possibly larger efficiency of our scheme since weaker focusing fields reduce the oscillator stiffness of the cavity, and therefore, betatron frequency $\omega_{\beta}$ is reduced, which for oscillator systems leads to enhancement of resonant amplitude [28]. The effect on the wake by ponderomotive force of the harmonics due to increasing field strength $a_{0}$ is analyzed further in Sec. V. Figure 4(b) shows the electron spectra for all three cases at the gas target exit. The total injected charge of electrons accelerated to energy $>100 \mathrm{MeV}$ varies by $<0.1 \%$ when comparing nonresonant and two-color resonant cases, showing negligible effect of the harmonics on the overall electron injection.

In Fig. 5, we show normalized transverse momentum and $\gamma$ evolution of the most energetic electrons for the nonresonant and two-color cases. For an electron interacting with an electromagnetic wave in a vacuum, the normalized transverse momentum amplitude is limited by the value $\tilde{p}_{x}<a_{0}$. The peak amplitudes of harmonics satisfy $a_{1,2} \approx 1$, and we see that the amplitude of normalized momentum reaches $\tilde{p}_{x} \approx 100$ in the two-color case, while in the nonresonant case, we see that the natural betatron oscillations are limited to normalized transverse momentum of $\tilde{p}_{x} \approx 30$. This proves the presence of the resonance phenomenon. A more careful analysis showing the presence of nonlinear resonances is presented in Fig. 6, where we show the evolutions of laser frequencies seen by a representative electron, betatron frequency, and normalized transverse momentum of the electron for all three cases. The nonresonant case shows that Eq. (10) is not satisfied throughout the evolution, and there is no associated growth of transverse momentum amplitude. The single-color resonant case shows primary resonance $\omega_{1} \approx \omega_{\beta}$ and the associated transverse momentum growth between 4 and $5 \mathrm{~mm}$ from the entrance of the target. The two-color resonant case shows nonlinear resonances for both shifted frequencies seen by the electron $\omega_{1}, \omega_{2}$ with harmonic orders $n=1,2,3,4,6$. We see that the strongest resonant growth reaching $\tilde{p}_{x} \approx 90$ corresponds to simultaneous harmonic resonance when $3 \omega_{\beta} \approx \omega_{1}$ and $4 \omega_{\beta} \approx \omega_{2}$ around $4.8 \mathrm{~mm}$ from the entrance of the target.

\section{X-RAY RADIATION ANALYSIS}

To see the effect of two-color resonance on $\mathrm{x}$-ray radiation emitted by the accelerated electrons, we have calculated the on-axis photon flux for all three PIC cases according to the radiation integral [18]

$$
\begin{aligned}
\frac{\mathbf{d}^{2} W}{\mathbf{d} \Omega \mathbf{d} \omega}=\frac{e^{2}}{16 \pi^{3} \epsilon_{0} c} & \mid \int_{-\infty}^{\infty} \frac{\mathbf{n} \times((\mathbf{n}-\boldsymbol{\beta}) \times \dot{\boldsymbol{\beta}})}{(1-\mathbf{n} \cdot \boldsymbol{\beta})^{2}} \\
& \times\left.\exp \left[i \omega\left(1-\frac{\mathbf{n} \cdot \mathbf{R}}{c}\right)\right] d t\right|^{2},
\end{aligned}
$$

where $\mathbf{R}$ is the distance vector between the radiating electron and the observer, $\mathbf{n}$ is the normalized vector pointing from the electron toward the observer, and $\boldsymbol{\beta}=\mathbf{v} / c$ is the normalized velocity. We extracted the electron trajectories from the PIC simulation results to calculate the radiation emitted according to Eq. (13). The knowledge of acceleration of the particle $\dot{\boldsymbol{\beta}}$ is necessary, which was calculated by interpolating the individual particle trajectories with a time step sufficiently small to achieve numerical convergence in Eq. (13). The results are presented in Fig. 7. We observe enhancement in the single-color case. However, a larger enhancement of more than an order of magnitude in peak photon flux and up to two orders of magnitude enhancement in the hard x-ray region is present in the two-color case, when compared with nonresonant betatron $\mathrm{x}$-ray generation. The critical energy for the nonresonant case is calculated as $\hbar \omega_{c} \approx 25 \mathrm{keV}$, while for the two-color resonant case is $\hbar \omega_{c} \approx 31 \mathrm{keV}$. We see that the number of photons is enhanced almost uniformly across the whole energy spectrum, with a stronger boost in the hard $\mathrm{x}$-ray region.

Our results can be compared with previously published works focusing on betatron $\mathrm{x}$-ray enhancement mentioned in Sec. I, for example, by longitudinal and transverse density modulation in Ref. [21], where an order of magnitude enhancement was achieved in the hard $\mathrm{x}$-ray region and a factor of $\sim 2-3$ in peak photon flux. In Ref. [20], a shift of critical energy into the millielectronvolt range due to the resonant 


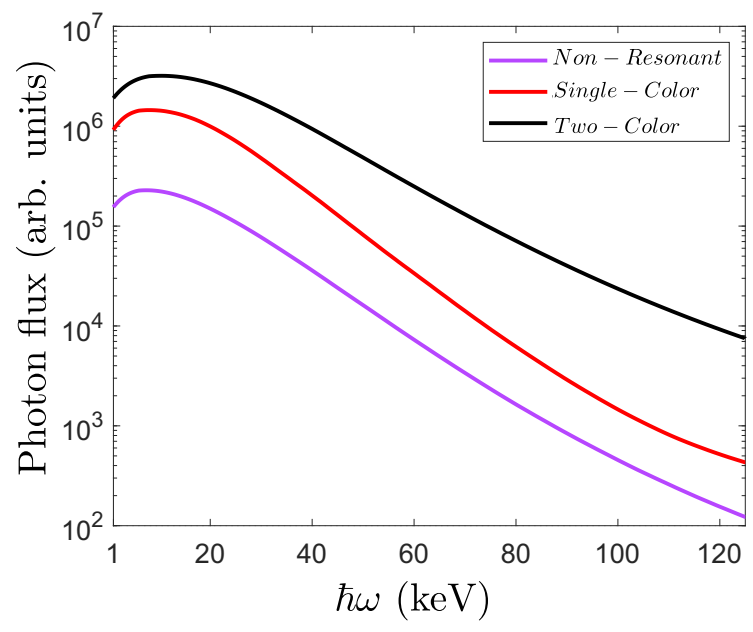

FIG. 7. On-axis photon flux of radiated $x$ rays in log scale. Nonresonant case (purple), single-color case (red), and the two-color case (black).

interaction of LWFA electrons with the laser tail of the driving laser was observed without peak photon flux enhancement. A peak photon flux enhancement due to resonant interaction with the laser tail from ionization injected electrons by a factor $\sim 3$ compared with results in Ref. [20] was reported in Ref. [27]. An order of magnitude enhancement was also achieved within the mildly nonlinear regime by steering the plasma wakefield driven by a laser with $a_{0}=1.6$ by introducing a slanted plasma target [25], where the betatron strength parameter $K$ in the reference betatron case without steering was $K \approx 2.1$. We note that this is more than an order of magnitude less than our reference nonresonant case with gigaelectronvolt range electrons reaching $K \approx 30$. We further note that the only requirement of our method is frequency conversion of the LWFA-driving laser into its harmonics, implying its potential compatibility with various plasma targets.

As mentioned in Sec. IV, the interacting laser field strengths $a_{i}$ cannot be too strong since the ponderomotive force scales as $\sim \nabla a^{2}$, which adversely affects wake evolution, resulting in poor electron injection and acceleration. To show this in terms of radiated $\mathrm{x}$ rays, we performed four different PIC simulations with the same parameters as in the two-color resonant case discussed earlier, except we did not include the SH and only kept the TH overlapping with the plasma sheath. In Fig. 8, we see the radiated energy where the fundamental laser acts as a LWFA driver, and the TH induces betatron resonance in oscillating electrons. We have varied the field strength of the TH for values $a_{0}=0.4,0.7,1,1.5$. The results show that x-ray photon flux starts dropping when $a_{0}$ approaches and surpasses the value $a_{0} \approx 1$, indicating modified wake dynamics by ponderomotive force and inefficient resonant oscillations. We note, however, that even in this inefficient case where $a_{0}=1.5$, the peak photon flux is still a factor of 2.5 larger than the standard betatron $\mathrm{x}$-ray generation denoted above as the nonresonant case. We see that, for the settings presented above, the value of $a_{2}=0.7$ is optimized for efficient radiation generation.

Lastly, we evaluate the practical energy efficiency of the two-color scheme. Let us consider a $7 \mathrm{~J}$ energy laser pulse

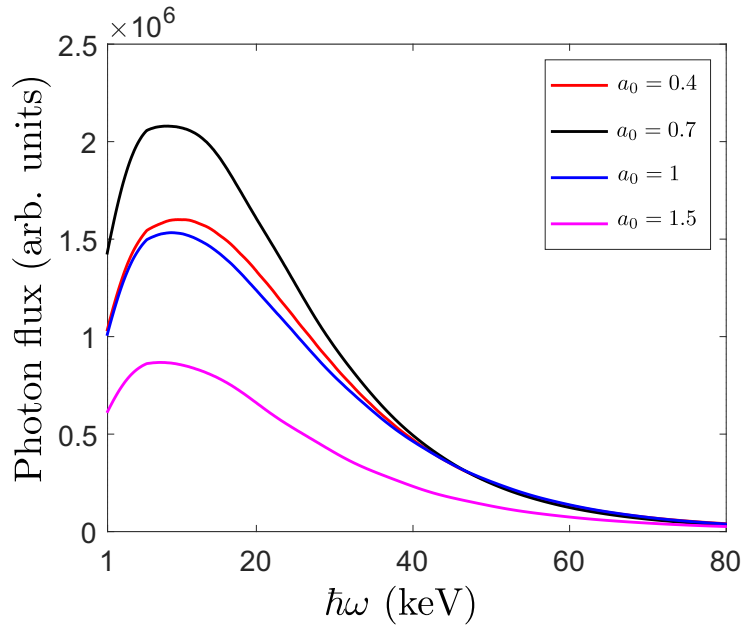

FIG. 8. On-axis photon flux of betatron $\mathrm{x}$ rays in linear scale showing decrease in radiated energy due to perturbation of the wake through increasing ponderomotive force of the third harmonic (TH). The laser fields present in the particle-in-cell (PIC) simulation are only the fundamental laser wakefield acceleration (LWFA) driver with wavelength $\lambda_{d}=808 \mathrm{~nm}$ and the TH with $\omega_{2}$.

having the same spatial and temporal parameters as discussed for the LWFA driver with fundamental wavelength $\lambda_{d}=808 \mathrm{~nm}$. This results in the field strength $a_{0} \approx 6$. We denote this case nonresonant, $7 \mathrm{~J}$. If we consider splitting off $1.9 \mathrm{~J}$ laser energy from the main $7 \mathrm{~J}$ beam and converting it to the $\mathrm{SH}$ and $\mathrm{TH}$ with conversion efficiency $\sim 11 \%$, where we take into consideration both parametric efficiency and other losses as discussed in Sec. VI, we may obtain the SH and TH with respective pulse energies of $\sim 0.2 \mathrm{~J}$, which corresponds to the spatial and temporal pulse parameters and field strengths of the harmonics as presented in the two-color resonant case. Here, we denote this case two-color, 5.5 J. In Fig. 9, we show that the photon flux of the second case with $5.5 \mathrm{~J}$ in total laser energy divided among the $\omega_{d}(5.1 \mathrm{~J}), \omega_{1}(0.2 \mathrm{~J})$, and $\omega_{2}(0.2 \mathrm{~J})$ is still significantly larger than for the case with the whole

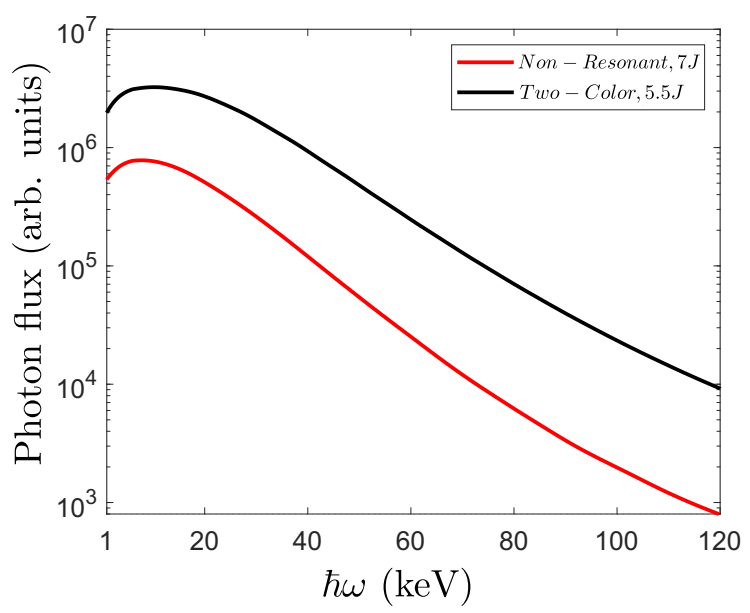

FIG. 9. On-axis photon flux of betatron $\mathrm{x}$ rays in log scale showing energy efficiency of the two-color betatron resonant x-ray generation. 


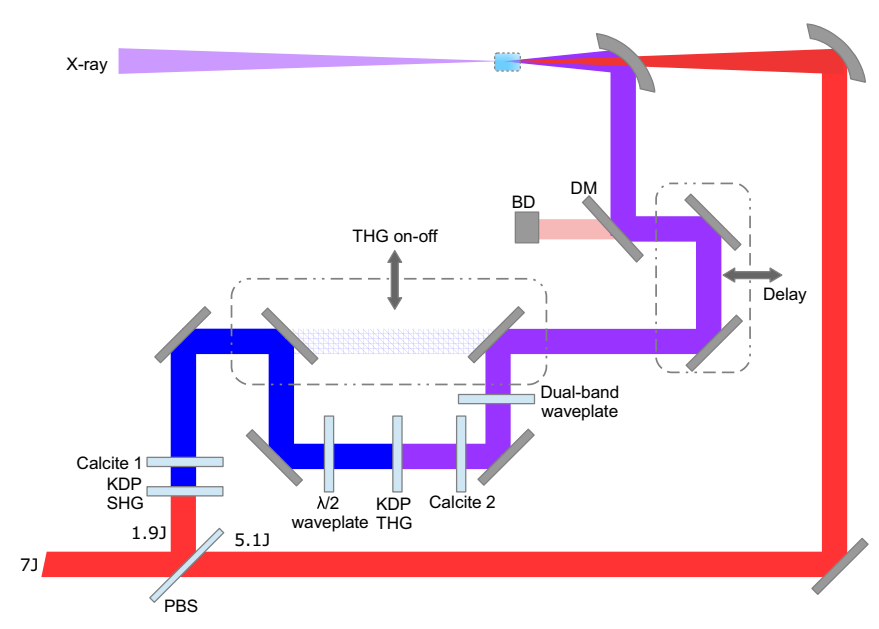

FIG. 10. Experimental setup design for realization of two-color resonant betatron $\mathrm{x}$-ray generation.

$7 \mathrm{~J}$ of energy in the LWFA driver $\omega$. This result indicates that the enhancement from two-color betatron resonance is highly efficient, and even when considering losses of $1.5 \mathrm{~J}$, we still get almost an order of magnitude enhancement in peak on-axis photon flux and more than an order of magnitude enhancement in the hard x-ray region. This shows that the enhancement from two-color betatron resonance cannot be reasonably replaced by boosting field strength $a_{0}$ in standard betatron x-ray generation. Crucially, we also point out that the nonresonant $7 \mathrm{~J}$ case results in $21 \%$ more injected total charge than the $5.5 \mathrm{~J}$ two-color case; however, not even this fact offsets the strong photon flux enhancement due to transverse momentum nonlinear resonances.

\section{EXPERIMENTAL SETUP DESIGN}

To complement our theoretical results, we propose the experimental setup, shown in Fig. 10, for realization of two-color resonant betatron $\mathrm{x}$-ray generation. Standard betatron $\mathrm{x}$-ray generation involves a single ultraintense $I \geqslant$ $10^{18} \mathrm{Wcm}^{-2}$ femtosecond laser pulse from a Ti:sapphire laser system centered at $808 \mathrm{~nm}$ and either a gas cell or a gas jet, where the gas is usually helium with a small percentage of some noble gas added to trigger ionization injection and achieve greater beam stabilization [40]. The advanced betatron scheme presented here requires the generation of the $\mathrm{SH}$ and $\mathrm{TH}$ of the fundamental $808 \mathrm{~nm}$ wavelength with pulse energy $\sim 200 \mathrm{~mJ}$ and pulse duration $\sim 50$ fs. Frequency tripling can be achieved directly in materials with strong third-order susceptibility with conversion efficiencies up to $6 \%$ [41]. However, due to higher conversion efficiency, a more commonly used method is of sum-frequency generation, which benefits from high values of second-order nonlinearity in crystals [42]. For Ti:sapphire frequency conversion, the widely used crystals for both frequency doubling and tripling are $\mathrm{BBO}$ and KDP [43]. For pulse duration of tens of femtoseconds, BBO crystals have the advantage over KDP in an almost six-times-higher effective nonlinear coefficient [44,45], but its spectral acceptance caused by group delay dispersion is roughly three times worse than in KDP crystals. The damage threshold for BBO crystals is on the order of hundreds of gigawatts per square centimeter for femtosecond pulses [46]. Combined with the difficulties involved in manufacturing of large aperture crystals, it is almost impossible to use BBO for few-joule femtosecond pulses.

To relate to the theoretical results, a $7 \mathrm{~J}, 28$ fs FWHM infrared (IR) laser pulse produced from a Ti:sapphire laser system is considered in the experimental setup; therefore, KDP crystals are considered further. The necessity of delay control between the IR LWFA driver and its harmonics leads to a two-arm experimental setup, where one arm has variable time delay. This requires micrometer-precise spatial synchronization and stability of the two differently focused beams [47]. In the proposed setup, a pellicle beamsplitter extracts a $1.9 \mathrm{~J}$ portion of the beam into the harmonic generation arm without any changes in the transmitted beam due to its thinness. The first KDP is a 1-mm-thick type-1 crystal used for efficient $\mathrm{SH}$ generation (SHG). The efficiency for hundreds of femtosecond pulses can reach as high as $80 \%$, as discussed in Ref. [48]. However, we consider pulse lengths on the order of $\sim 50$ fs in our PIC simulations. Thus, the efficiency will be reduced due to group velocity dispersion. Calcite 1 is negative uniaxial crystal used for compensation of group delay between IR and SH pulses originated in KDP as well as in the half-waveplate. The temporal overlap of IR and SH pulses is crucial in the second KDP crystal used for $\mathrm{TH}$ generation (THG). A thin, zero-order half-waveplate for IR wavelength also serves as a $\lambda$ waveplate for the $\mathrm{SH}$, which assures parallel polarization for both the IR and $\mathrm{SH}$ to ensure THG type-1 phase matching in the second KDP crystal. This is to exploit the higher conversion efficiency of type- 1 over type- 2 THG $[42,43]$. THG in this configuration can lead to conversion efficiency up to $11 \%$, as discussed in Ref. [43]. Calcite 2 is used (similarly to Calcite 1) for group delay compensation between the residual $\mathrm{SH}$ and the generated TH. A dual-band waveplate is optimized as $\lambda / 2$ for the $\mathrm{SH}$ and $\lambda$ for the $\mathrm{TH}$ to rotate polarizations of the $\mathrm{SH}$ and $\mathrm{TH}$ to be parallel, it can be easily moved out to switch to a configuration where the TH is perpendicular to the SH. Group delay from the dual-band waveplate is also compensated by Calcite 2.

The proposed setup has the possibility to easily switch off THG (THG on-off) with a motorized linear stage and therefore easily go between single-color, two-color, or two-color with a single harmonic, where resonance is achieved due to a long-pulse LWFA-driving laser and a SH with tunable laser polarizations. A dichroic mirror is optimized for reflectance of the $\mathrm{TH}$ and $\mathrm{SH}$ and therefore transmits the residual IR pulse into the beam dump. According to discussed efficiencies, this system should be capable of delivering three-color interaction into the target composed of $5.1 \mathrm{~J} \mathrm{IR}, 200 \mathrm{~mJ} \mathrm{SH}$, and $200 \mathrm{~mJ}$ TH ultrashort pulses. For higher energy laser systems, even greater range of optimization is possible with more energy in the IR beam and possibly the harmonics as well, if crystals of large enough aperture are available. The advantages of this setup are the full control over the laser polarizations and the delays between the IR and $\mathrm{SH}$ as well as between the $\mathrm{SH}$ and $\mathrm{TH}$ and separate focusing for the harmonics through an off-axis parabola with a hole. The conversion efficiency can be tuned with crystal angle tuning. 


\section{CONCLUSIONS}

In summary, we have presented theory and numerical results exploring two-color nonlinear resonances in betatron oscillations and an experimental setup design for realization of our scheme. We have shown that the dynamics of relativistic electrons reduce to nonlinear oscillations with twofrequency forcing, which causes the emergence of nonlinear resonances in transverse momentum. PIC simulations for experimentally accessible parameters reveal two-color betatron resonance behavior beyond the paraxial approximation, showing strong enhancement of electron transverse momentum. The radiation emitted by electrons undergoing such resonant oscillations is enhanced by more than an order of magnitude in peak photon flux and up to two orders of magnitude in the hard $\mathrm{x}$-ray region when compared with standard betatron $\mathrm{x}$-ray generation.

\section{ACKNOWLEDGMENTS}

This paper was supported by the project ADONIS (CZ.02.1.01/0.0/0.0/16_019/0000789) and by the project HiFi (CZ.02.1.01/0.0/0.0/15_003/0000449) from the European Regional Development Fund and the project LM2015065 as part of targeted support of Large Infrastructures of the Ministry of Education, Youth and Sports of the Czech Republic. This work was supported by the Charles University grant SVV2021-260590.
[1] T. Tajima and J. M. Dawson, Laser Electron Accelerator, Phys. Rev. Lett. 43, 267 (1979).

[2] A. J. Gonsalves et al., Petawatt Laser Guiding and Electron Beam Acceleration to $8 \mathrm{Gev}$ in a Laser-Heated Capillary Discharge Waveguide, Phys. Rev. Lett. 122, 084801 (2019).

[3] A. Pukhov and J. Meyer-ter Vehn, Laser wake field acceleration: the highly non-linear broken-wave regime, Appl. Phys. B 74, 355 (2002).

[4] W. Lu, C. Huang, M. Zhou, W. B. Mori, and T. Katsouleas, Nonlinear Theory for Relativistic Plasma Wakefields in the Blowout Regime, Phys. Rev. Lett. 96, 165002 (2006).

[5] S. Kiselev, A. Pukhov, and I. Kostyukov, X-Ray Generation in Strongly Nonlinear Plasma Waves, Phys. Rev. Lett. 93, 135004 (2004).

[6] A. Rousse, K. T. Phuoc, R. Shah, A. Pukhov, E. Lefebvre, V. Malka, S. Kiselev, F. Burgy, J.-P. Rousseau, D. Umstadter, and D. Hulin, Production of a keV X-Ray Beam from Synchrotron Radiation in Relativistic Laser-Plasma Interaction, Phys. Rev. Lett. 93, 135005 (2004).

[7] S. Corde, K. Ta Phuoc, G. Lambert, R. Fitour, V. Malka, A. Rousse, A. Beck, and E. Lefebvre, Femtosecond x rays from laser-plasma accelerators, Rev. Mod. Phys. 85, 1 (2013).

[8] S. Fourmaux, E. Hallin, U. Chaulagain, S. Weber, and J. Kieffer, Laser-based synchrotron x-ray radiation experimental scaling, Opt. Express 28, 3147 (2020).

[9] F. Albert, A. Thomas, S. Mangles, S. Banerjee, S. Corde, A. Flacco, M. Litos, D. Neely, J. Vieira, Z. Najmudin, R. Bingham, C. Joshi, and T. Katsouleas, Laser wakefield accelerator based light sources: potential applications and requirements, Plasma Phys. Controlled Fusion 56, 084015 (2014).

[10] J. Wenz, S. Schleede, K. Khrennikov, M. Bech, P. Thibault, M. Heigoldt, F. Pfeiffer, and S. Karsch, Quantitative X-ray phasecontrast microtomography from a compact laser-driven betatron source, Nat. Commun. 6, 7568 (2015).

[11] U. Chaulagain, K. Bohacek, M. Kozlova, J. Nejdl, M. Krus, V. Horny, B. Mahieu, and K. Ta-Phuoc, X-ray phase contrast imaging of biological samples using a betatron $\mathrm{x}$-ray source generated in a laser wakefield accelerator, in Laser Acceleration of Electrons, Protons, and Ions IV (International Society for Opti and Photonics, Bellingham, WA, 2017), Vol. 10240, p. 1024014.

[12] J. C. Wood et al., Ultrafast imaging of laser driven shock waves using betatron $\mathrm{x}$-rays from a laser wakefield accelerator, Sci. Rep. 8, 11010 (2018).
[13] B. Mahieu, N. Jourdain, K. T. Phuoc, F. Dorchies, J.-P. Goddet, A. Lifschitz, P. Renaudin, and L. Lecherbourg, Probing warm dense matter using femtosecond x-ray absorption spectroscopy with a laser-produced betatron source, Nat. Commun. 9, 3276 (2018).

[14] J.-N. Gruse et al., Application of compact laser-driven accelerator $\mathrm{x}$-ray sources for industrial imaging, Nucl. Instrum. Methods Phys. Res. Sect. A 983, 164369 (2020).

[15] B. Kettle et al., Single-Shot Multi-Kev X-Ray Absorption Spectroscopy Using an Ultrashort Laser-Wakefield Accelerator Source, Phys. Rev. Lett. 123, 254801 (2019).

[16] E. Esarey, B. A. Shadwick, P. Catravas, and W. P. Leemans, Synchrotron radiation from electron beams in plasma-focusing channels, Phys. Rev. E 65, 056505 (2002).

[17] S. Fourmaux, S. Corde, K. Ta Phuoc, P. M. Leguay, S. Payeur, P. Lassonde, S. Gnedyuk, G. Lebrun, C. Fourment, V. Malka, S. Sebban, A. Rousse, and J. C. Kieffer, Demonstration of the synchrotron-type spectrum of laser-produced betatron radiation, New J. Phys. 13, 033017 (2011).

[18] J. D. Jackson, Classical Electrodynamics (John Wiley \& Sons, New York, 1999).

[19] B. Lei, J. Wang, V. Kharin, M. Zepf, and S. Rykovanov, $\gamma$-Ray Generation from Plasma Wakefield Resonant Wiggler, Phys. Rev. Lett. 120, 134801 (2018).

[20] S. Cipiccia et al., Gamma-rays from harmonically resonant betatron oscillations in a plasma wake, Nat. Phys. 7, 867 (2011).

[21] M. Kozlova, I. Andriyash, J. Gautier, S. Sebban, S. Smartsev, N. Jourdain, U. Chulagain, Y. Azamoum, A. Tafzi, J.-P. Goddet, K. Oubrerie, C. Thaury, A. Rousse, and K. Ta Phuoc, Hard X Rays from Laser-Wakefield Accelerators in Density Tailored Plasmas, Phys. Rev. X 10, 011061 (2020).

[22] Y. Ma, L. Chen, D. Li, W. Yan, K. Huang, M. Chen, Z. Sheng, K. Nakajima, T. Tajima, and J. Zhang, Generation of femtosecond $\gamma$-ray bursts stimulated by laser-driven hosing evolution, Sci. Rep. 6, 30491 (2016).

[23] S. G. Rykovanov, C. B. Schroeder, E. Esarey, C. G. R. Geddes, and W. P. Leemans, Plasma Undulator Based on Laser Excitation of Wakefields in a Plasma Channel, Phys. Rev. Lett. 114, 145003 (2015).

[24] X.-L. Zhu, M. Chen, S.-M. Weng, T.-P. Yu, W.-M. Wang, F. He, Z.-M. Sheng, P. McKenna, D. A. Jaroszynski, and J. Zhang, Extremely brilliant geV $\gamma$-rays from a two-stage laser-plasma accelerator, Sci. Adv. 6, eaaz7240 (2020). 
[25] C. Yu, J. Liu, W. Wang, W. Li, R. Qi, Z. Zhang, Z. Qin, J. Liu, M. Fang, K. Feng, Y. Wu, L. Ke, Y. Chen, C. Wang, Y. Xu, Y. Leng, C. Xia, R. Li, and Z. Xu, Enhanced betatron radiation by steering a laser-driven plasma wakefield with a tilted shock front, Appl. Phys. Lett. 112, 133503 (2018).

[26] A. Pukhov, Z.-M. Sheng, and J. Meyer-ter Vehn, Particle acceleration in relativistic laser channels, Phys. Plasmas 6, 2847 (1999).

[27] K. Huang, Y. F. Li, D. Z. Li, L. M. Chen, M. Z. Tao, Y. Ma, J. R. Zhao, M. H. Li, M. Chen, M. Mirzaie, N. Hafz, T. Sokollik, Z. M. Sheng, and J. Zhang, Resonantly enhanced betatron hard x-rays from ionization injected electrons in a laser plasma accelerator, Sci. Rep. 6, 27633 (2016).

[28] A. H. Nayfeh and D. T. Mook, Nonlinear Oscillations (John Wiley \& Sons, New York, 2008).

[29] I. Kostyukov, A. Pukhov, and S. Kiselev, Phenomenological theory of laser-plasma interaction in "bubble" regime, Phys. Plasmas 11, 5256 (2004).

[30] A. Pukhov, S. Gordienko, S. Kiselev, and I. Kostyukov, The bubble regime of laser-plasma acceleration: monoenergetic electrons and the scalability, Plasma Phys. Controlled Fusion 46, B179 (2004).

[31] B.-S. Xie, H.-C. Wu, H. Wang, N.-Y. Wang, and M. Yu, Analysis of the electromagnetic fields and electron acceleration in the bubble regime of the laser-plasma interaction, Phys. Plasmas 14, 073103 (2007).

[32] B. Liu, H. Y. Wang, J. Liu, L. B. Fu, Y. J. Xu, X. Q. Yan, and X. T. He, Generating Overcritical Dense Relativistic Electron Beams via Self-Matching Resonance Acceleration, Phys. Rev. Lett. 110, 045002 (2013).

[33] L. D. Landau and E. M. Lifshitz, Mechanics (Pergamon Press, Oxford, 1976).

[34] T. D. Arber, K. Bennett, C. S. Brady, A. Lawrence-Douglas, M. G. Ramsay, N. J. Sircombe, P. Gillies, R. G. Evans, H. Schmitz, A. R. Bell, and C. P. Ridgers, Contemporary particle-in-cell approach to laser-plasma modelling, Plasma Phys. Controlled Fusion 57, 113001 (2015).

[35] R. Lehe, C. Thaury, E. Guillaume, A. Lifschitz, and V. Malka, Laser-plasma lens for laser-wakefield accelerators, Phys. Rev. ST Accel. Beams 17, 121301 (2014).

[36] F. Li, K. G. Miller, X. Xu, F. S. Tsung, V. K. Decyk, W. An, R. A. Fonseca, and W. B. Mori, A new field solver for modeling of relativistic particle-laser interactions using the particle-incell algorithm, Comput. Phys. Commun. 258, 107580 (2021).

[37] L.-L. Yu, E. Esarey, C. B. Schroeder, J.-L. Vay, C. Benedetti, C. G. R. Geddes, M. Chen, and W. P. Leemans,
Two-Color Laser-Ionization Injection, Phys. Rev. Lett. 112, 125001 (2014).

[38] C. McGuffey, A. G. R. Thomas, W. Schumaker, T. Matsuoka, V. Chvykov, F. J. Dollar, G. Kalintchenko, V. Yanovsky, A. Maksimchuk, K. Krushelnick, V. Yu. Bychenkov, I. V. Glazyrin, and A. V. Karpeev, Ionization Induced Trapping in a Laser Wakefield Accelerator, Phys. Rev. Lett. 104, 025004 (2010)

[39] A. Golovanov and I. Y. Kostyukov, Bubble regime of plasma wakefield in $2 d$ and $3 d$ geometries, Phys. Plasmas 25, 103107 (2018).

[40] A. Döpp, B. Mahieu, A. Lifschitz, C. Thaury, A. Doche, E. Guillaume, G. Grittani, O. Lundh, M. Hansson, J. Gautier, M. Kozlova, J. P. Goddet, P. Rousseau, A. Tafzi, V. Malka, A. Rousse, S. Corde, and K. Ta Phuoc, Stable femtosecond $\mathrm{X}$-rays with tunable polarization from a laser-driven accelerator, Light: Sci. Appl. 6, e17086 (2017).

[41] P. S. Banks, M. D. Feit, and M. D. Perry, High-intensity thirdharmonic generation, JOSA B 19, 102 (2002).

[42] W. Yan-Ling, Z. Xu-Gui, W. Hong, and D. Liang-En, Efficient collinear frequency tripling of femtosecond laser with compensation of group velocity delay, Chin. Phys. B 18, 4308 (2009).

[43] Y. Nabekawa, K. Kondo, N. Sarukura, K. Sajiki, and S. Watanabe, Terawatt KrF/Ti:sapphire hybrid laser system, Opt. Lett. 18, 1922 (1993).

[44] R. C. Eckardt and R. L. Byer, Measurement of nonlinear optical coefficients by phase-matched harmonic generation, in Inorganic Crystals for Optics, Electro-Optics, and Frequency Conversion (International Society for Optics and Photonics, Bellingham, 1991), Vol. 1561, pp. 119-127.

[45] G. Bhar, A. Chaudhary, P. Kumbhakar, A. Rudra, and S. Sabarwal, A comparative study of laser-induced surface damage thresholds in BBO crystals and effect of impurities, Opt. Mater. (Amsterdam) 27, 119 (2004).

[46] V. Krylov, J. Gallus, U. Wild, A. Kalintsev, and A. Rebane, Femtosecond noncollinear and collinear parametric generation and amplification in BBO crystal, Appl. Phys. B 70, 163 (2000).

[47] S. Li, G. Li, Q. Ain, M. S. Hur, A. C. Ting, V. V. Kulagin, C. Kamperidis, and N. A. Hafz, A laser-plasma accelerator driven by two-color relativistic femtosecond laser pulses, Sci. Adv. 5, eaav7940 (2019).

[48] M. Aoyama, T. Harimoto, J. Ma, Y. Akahane, and K. Yamakawa, Second-harmonic generation of ultra-high intensity femtosecond pulses with a KDP crystal, Opt. Express 9, 579 (2001). 